\title{
Educar a niñas y niños de clase media en Santiago: \\ capital cultural y segregación socioterritorial en la formación de mercados locales de educación
}

Joel Stillerman. Grand Valley State University, Allendale, Michigan, Estados Unidos.

RESUMEN | Muchos investigadores han estudiado en forma paralela la segregación residencial y la educacional, sin considerar cómo se relacionan ambos procesos. Este artículo explora la forma en que los padres de clase media eligen colegios en Nuñoa y La Florida (Santiago, Chile). Nuñoa es una comuna de clase media alta con una gran dotación de colegios particulares pagados; La Florida, en tanto, contiene una clase media de formación más reciente, una tasa de pobreza más alta y una gran cantidad de colegios subvencionados. Este artículo, basado en entrevistas semiestructuradas con 77 padres, revela que, en Nuńoa, los padres eligen entre colegios particulares pagados y descartan la opción de enviar a sus hijos a colegios municipales o subvencionados. $\mathrm{Al}$ contrario, los padres de La Florida eligen colegios subvencionados como una forma de evitar los peligros que perciben en los colegios municipales. Estos resultados tienen significativas implicancias para los debates sobre la segregación residencial y educacional.

PALABRAS CLAVE | capital cultural, cultura urbana, segregación.

ABSTRACT | Many researchers have studied residential and educational segregation processes without considering the relationship between them. This article explores how middle class parents select schools in the municipalities of Nuñoa and La Florida (Santiago, Chile). Nuñoa is an upper middle-class municipality with a large number of elite private schools; while La Florida has a newer middle class, a higher poverty rate, and many charter schools. Based on in-depth interviews with 77 parents, this article finds that in Nuñoa parents choose elite private schools and reject charter or public schools. In contrast, parents in La Florida select charter schools as a way to avoid the dangers associated with public schools. The results have important implications for debate on residential and school segregation.

KEY WORDS | cultural capital, urban culture, segregation. 


\section{Introducción}

El sistema de educación chileno ha provocado un prolongado debate entre académicos, funcionarios del gobierno y activistas estudiantiles. Se ha argumentado en torno a la implementación, a partir de 1981, de un sistema de subvención pública (vouchers) a colegios particulares y públicos cuyo objetivo es permitir a las familias elegir el colegio más adecuado para sus hijos. En teoría, la competencia entre colegios para captar alumnos obligaría a todos los colegios a mejorar la calidad de la educación que ofrecen. Sin embargo, muchos autores plantean que la educación escolar no ha mejorado en forma apreciable, y que el sistema de subvención ha intensificado la segregación educacional. En forma paralela, diversos investigadores han estudiado los efectos negativos de la segregación residencial para las oportunidades en el mercado laboral, el acceso a servicios y la vida cotidiana de personas en situación de pobreza. Mientras algunos autores enfatizan que la oferta local de colegios en cada barrio o comuna afecta las opciones educativas de las familias, pocos han estudiado la relación entre la segregación residencial y la educacional.

Este artículo propone llenar este vacío a través de una aproximación hacia las estrategias de elección de colegios de padres de distintas fracciones de las clases medias en Santiago, Chile. A partir de 68 entrevistas semiestructuradas con 77 apoderados cuyos hijos estudian en las comunas de Nunñoa y La Florida (y sus alrededores), se pretende entender cómo el universo de opciones educativas en cada comuna afecta el proceso de elección de colegio para sus residentes.

Las clases medias en Chile han generado mucho interés entre los investigadores en los últimos años. Los especialistas han registrado cambios en su tamaño y estructura (León \& Martínez, 2007; Barozet \& Fierro, 2011), en el papel del capital social como recurso de movilidad ascendente (Barozet, 2006), en la evolución de las identidades individuales y colectivas (Ariztía, 2009; Méndez, 2008; Méndez \& Barozet, 2012) y en la disposición de miembros de la clase media y la clase alta a interactuar con personas pobres (Sabatini, Rasse, Mora \& Brain, 2012; Rasse, 2015).

Esta investigación propone aplicar algunas ideas sobre la segregación residencial al caso de la elección de escuelas de parte de familias de clase media. También hace un aporte a la investigación internacional sobre educación y desigualdad, que se ha enfocado principalmente en los Estados Unidos (Lareau, 2003) y Europa (Ball, Bowe \& Gerwitz, 1995; Ball \& Vincent, 1998; Bourdieu, 1997, 1984; Bourdieu \& Passeron, 1990; Raveuda \& Van Zanten, 2007).

Los resultados de esta investigación muestran importantes diferencias en la oferta de establecimientos educacionales en las dos comunas y la forma en que estas diferencias afectan las decisiones de los padres. Nuñoa es una comuna principalmente de clase media alta con muchos colegios particulares pagados. En este contexto, los entrevistados de clase media alta descartan los colegios particulares subvencionados y los municipales como opciones para sus hijos. Se preocupan de contrastar su elección de un colegio que da una educación integral para sus hijos, con las opciones de otros padres que eligen colegios "elitistas" o "exitistas"; en esto, como en otros aspectos, se comparan con la elite. Además, muchos de estos adultos estudiaron 
en colegios particulares o liceos públicos "emblemáticos"1 e incluso varios son exalumnos de los establecimientos donde hoy estudian sus hijos. Con sus opciones actuales, intentan continuar una tradición de educación que experimentaron en su infancia. En contraste, aunque varios de los entrevistados en La Florida también son de clase media alta, tienden a elegir colegios subvencionados para sus hijos, porque hay poca oferta de colegios particulares $y$, en general, encuentran que estos ofrecen una educación de baja calidad. En La Florida, las familias de clase media baja prefieren colegios cercanos a sus casas, que protegen a sus hijos de las "malas" influencias de familias pobres. En esta comuna, la fuga de familias de clase media de los colegios municipales hacia los subvencionados se hace evidente.

Los resultados señalados constituyen un aporte a los debates sobre la segregación residencial y educativa. La discusión sobre la segregación residencial se enfoca en sus efectos en las oportunidades laborales, acceso a servicios y vida cotidiana de distintas clases sociales. Este artículo señala que la segregación de los colegios entre comunas refleja e intensifica los efectos de la segregación residencial y, a su vez, afecta las oportunidades laborales de personas de distintas clases sociales. Además, muestra que la composición socioeconómica de una comuna incide en la forma en que las acciones de sus residentes contribuyen a la reproducción de la segregación educacional. Los residentes de comunas de mayores ingresos evitan el sistema público y subvencionado por completo; los residentes de clase media en comunas mixtas a veces matriculan a sus hijos en colegios municipales en un primer momento, pero tienden a cambiarlos a colegios subvencionados. Los resultados de esta investigación también hacen otro aporte a los debates sobre la segregación educacional. Algunos autores se han concentrado en cómo los colegios particulares y subvencionados excluyen a estudiantes pobres o discapacitados, mientras otros se han enfocado en cómo los padres de la clase media evitan los colegios municipales. En cambio, este artículo muestra que la oferta local de colegios afecta las opciones de los padres; pero, a su vez, los padres contribuyen a la segregación a través de sus opciones educativas.

$\mathrm{El}$ argumento se desarrolla en las siguientes etapas. Se comienza con una revisión de la literatura sobre la segregación residencial; se prosigue con un resumen de la literatura sobre la segregación educacional, tanto en Chile como a nivel internacional; luego, se describe el diseño metodológico del estudio. Finalmente se discuten los resultados del estudio y se concluye con una consideración sobre algunas implicaciones del análisis.

\section{La segregación residencial y las clases medias}

El debate sobre la segregación residencial en Santiago se ha enfocado en los efectos de la segregación de gran y pequeña escala. La segregación de gran escala se refiere a

1 En Chile se denomina liceos "emblemáticos" a aquellos establecimientos educacionales públicos (estatales) tradicionales, de gran prestigio y reconocida excelencia. Sobre estructura del sistema educativo en Chile, puede verse: Organización de Estados Iberoamericanos para la Educación, la Ciencia y la Cultura (OEI). (1993). Sistemas educativos nacionales - Chile. Informe OEI-Ministerio 1993. Cap. 4: "Estructura del sistema educacional”, p. 3. En http://www.oei.es/quipu/chile/ CHIL04.PDF [N. de E.] 
programas estatales que promovieron la construcción de viviendas sociales en áreas periféricas de las ciudades chilenas. Estas políticas buscaron maximizar la cantidad de viviendas construidas y, por tanto, optaron por edificarlas en lugares con bajos valores de suelo. Los residentes de estas viviendas sufrieron los efectos negativos de la concentración espacial de la pobreza, y la ubicación de los conjuntos habitacionales de interés social hizo difícil el acceso al trabajo y servicios para sus ocupantes (Sabatini \& Cáceres, 2004; Sabatini, Cáceres \& Cerda, 2001).

La segregación residencial de pequeña escala ha provocado distintas interpretaciones. En las últimas décadas, la construcción de condominios cerrados para grupos de ingresos medios y altos en comunidades pobres ha creado la posibilidad de mayor contacto cotidiano entre personas de distintas clases sociales. Al respecto, algunos investigadores plantean que los residentes de los barrios cerrados tienden a evitar el contacto con los antiguos residentes pobres que viven al lado de los condominios (Márquez \& Pérez, 2008). En contraste, Cáceres y Sabatini (2004) plantean que la construcción de los condominios en áreas pobres permite la integración funcional de residentes de distintas clases sociales y promueve el mejoramiento de los servicios en los barrios respectivos. Sabatini et al. (2012) encontraron que miembros de la clase media están aceptando vivir cerca a familias pobres, siempre que estas no se encuentren en su entorno inmediato. Según Rasse (2015), las personas ricas y las pobres que viven en el mismo entorno logran desarrollar un sentido de pertenencia a su barrio, siempre y cuando no se den problemas de violencia y las brechas de ingreso entre los dos grupos no sean demasiado grandes. Este artículo hace un aporte a la discusión más reciente, porque estudia hasta qué punto las familias de clase media están dispuestas a enviar a sus hijos a colegios con niños pobres, uno de los contextos más difíciles de integración social, según Sabatini et al. (2012).

\section{Padres, colegios y desigualdad}

Distintos investigadores han explorado cómo el sistema educativo contribuye a la reproducción de la estructura de clases. Inspirados por las investigaciones de Pierre Bourdieu, han estudiado la manera en que la introducción de elementos de mercado en los sistemas educativos en los Estados Unidos y Europa ha incidido en la desigualdad en el acceso a la educación básica y media entre los niños de las clases medias y la clase trabajadora. Al respecto, lo que las investigaciones demuestran es que los sistemas de "elección" favorecen a las familias que ya están privilegiadas en el campo educativo y, por tanto, aumentan las desigualdades entre la clase trabajadora y la clase media.

Siguiendo a Pierre Bourdieu, varios autores se enfocan en las decisiones de las familias respecto de la elección de colegios para sus hijos, y en la relación de dichas decisiones con la clase social y los discursos culturales específicos de esos grupos. Bourdieu (1984; 1997) y Bourdieu y Passeron (1990) plantean que niñas y niños de distintas clases sociales entran al colegio con diferentes ventajas y desventajas basadas en su capital lingüístico y familiaridad con elementos de alta cultura, aspectos que son valorizados por el sistema educativo. Los conocimientos y valores de los padres de la clase alta se acercan a los valores de los colegios y las universidades. Por tanto, 
sus hijos e hijas están mejor preparados que miembros de las otras clases sociales para entrar a colegios y universidades de elite, que los preparan intelectualmente y les proveen de redes que sustentan su permanencia en la clase dominante.

Bourdieu $(1984 ; 1997)$ especifica dos modos de la adquisición de capital cultural: el modo doméstico y el modo académico. Plantea que las personas cuyos padres tienen altos niveles de educación parecen más cómodas y relajadas en la expresión de sus conocimientos, a los cuales han estado expuestos desde muy jóvenes. En este sentido, llegan "adelantados" al colegio, y su conocimiento parece más natural y valioso. El modo doméstico de adquisición de capital cultural también esconde el privilegio económico que constituye la base de su capital cultural, porque solo es posible si los niños tienen el tiempo de ocio para leer, estudiar música, etcétera.

En este contexto, Ball et al. (1995) examinan las diferencias entre los padres de la clase media y los de la clase trabajadora en relación con la elección de colegios en Inglaterra. Plantean que los padres de clase media se fijan en las reputaciones de colegios particulares de elite y colegios públicos subvencionados, intentan ubicar un colegio que calce con las características específicas de su hijo, buscan colegios con estudiantes de la misma clase social, tienen la capacidad para negociar la entrada de su hijo en su colegio preferido, y ven las consecuencias de sus decisiones para el desarrollo de su hijo en el largo plazo. Los padres de la clase trabajadora tienden a elegir colegios locales porque no presentan dificultades para el transporte de los niños al colegio, tienen miedo de enviarlos a a un lugar más lejano, necesitan que sus familiares cuiden a sus hijos y tienen un apego emocional con su barrio.

Ball y Vincent (1998) examinan cómo las redes sociales afectan las decisiones educativas de los padres en Inglaterra. En el proceso de identificar un colegio para sus hijos, todos los padres prefieren utilizar recomendaciones de personas cercanas (hot knowledge) antes que datos formales entregados por los colegios mismos o por el gobierno (cold knowledge).

Raveauda y Van Zanten (2007) han estudiado a padres de la clase media en Londres y París que intentan ser "buenos padres" y "buenos ciudadanos" a través de su elección de colegios públicos con un alto porcentaje de familias pobres o inmigrantes. Para negociar la tensión entre sus dos deseos, optan por rellenar los vacíos de la educación que reciben sus hijos a través de clases particulares, o intentan mejorar los colegios de sus hijos a través de un trabajo voluntario que apoya a los niños inmigrantes o a través de la promoción de su colegio a más familias de clase media. Por último, Ball (2003) comenta que en distintos países, familias de clase media usan sus capitales y capacidades para favorecer a sus hijos y así contribuyen a la reproducción de la segregación educacional.

La discusión sobre la educación básica y media en Chile se centra en las consecuencias de su reforma en 1981 bajo el régimen militar. El gobierno abrió la opción de crear colegios particulares subvencionados con el apoyo del fisco, y cambió el sistema de financiamiento y fiscalización de los colegios desde el nivel nacional al nivel municipal. Como resultado de las reformas, hubo un crecimiento impresionante de los colegios subvencionados. En 2010, el 52\% de los colegios eran subvencionados, $8 \%$ eran particulares y 40\%, municipales (Alves et al., 2015). La creación del sector subvencionado, más la ampliación de la educación municipal 
bajo los gobiernos de la Concertación (1990-2010), ha significado que para las generaciones jóvenes hay una cobertura casi universal en la educación básica y que aproximadamente $80 \%$ de la población ha terminado la educación media. En este sentido, hubo un crecimiento absoluto en el nivel de educación de la población. Sin embargo, el rendimiento académico a través del sistema educativo ha sido deficiente (Chumacero, Gómez \& Paredes, 2011; Contreras, Sepúlveda \& Barros, 2010).

La mayoría de los estudios en este ámbito compara la calidad de la educación entre los colegios municipales y los subvencionados. Al respecto, encuentran poca o nula diferencia de calidad entre ambos grupos según los resultados en las pruebas del Sistema de Medición de la Calidad de la Educación (simce) y la Prueba de Selección Universitaria (PSU). En la Región Metropolitana, los colegios subvencionados presentan una leve ventaja respecto de los municipales, y se acercan a la calidad de los particulares pagados en la enseñanza media. Sin embargo, estas ventajas pueden ser resultado de la selección de alumnos mediante el cobro de un alto precio de matrícula o el uso de exámenes de admisión que excluyen a estudiantes pobres, con un rendimiento académico limitado, con discapacidades y con problemas de comportamiento (cream skimming) (Contreras et al., 2010). Mizala y Torche (2012) encontraron que hay más estratificación socioeconómica y de rendimiento académico dentro del sector particular subvencionado que entre este y el municipal, mostrando con ello que las diferencias en resultados académicos reflejan el estatus socioeconómico de los padres en vez de la oferta pedagógica de los colegios. En 2008, el gobierno promulgó la Ley de Subvención Escolar Preferencial (SEP) para eliminar la selección de estudiantes en los colegios subvencionados, pero estos pueden negarse a participar en el programa. Si toman esta opción, pierden una subvención, pero no tienen que asumir los costos adicionales de enseñar a niños pobres o con discapacidades. Por tanto, $40 \%$ de los colegios subvencionados no participa en este programa; los colegios particulares no están regulados por el gobierno (Elacqua \& Santos, 2013). En mayo de 2015, la Presidenta Bachelet promulgó la Ley de Inclusión Escolar que prohíbe el lucro, el cobro de matrículas y la selección de alumnos colegios particulares subvencionados. Esta ley entró en vigencia al principios de 2016, pero aún no se sabe si logre reducir la desigualdad de acceso a la educación de calidad de alumnos que pertenecen a distintos grupos socioeconómicos (Vargas, 2015).

Aunque la reforma de 1981 fue diseñada para crear un mercado donde los "consumidores" podrían elegir el mejor producto, las investigaciones muestran que la calidad de la educación no ha mejorado como resultado de la entrada de colegios subvencionados. Thieme y Treviño (2013) indican que los apoderados eligen a un grupo socioeconómico más que los resultados académicos del colegio; Raczynski, Salinas, de la Fuente, Hernández y Lattz (2010) plantean que los apoderados eligen los colegios basándose en varios factores, pero solo los padres con mayores recursos están dispuestos a enviar a sus hijos a colegios más lejos de sus casas si tienen mayor calidad académica.

En el estudio cualitativo disponible más detallado sobre la elección de colegios en Chile, Raczynski et al. (2010) identifican diferencias importantes entre familias pobres cuyos niños estudian en colegios municipales y familias de clase media con 
hijos en colegios subvencionados. Los autores plantean que los padres eligen colegios a partir de su propio imaginario sobre los establecimientos, sus valores personales y sus aspiraciones. Algunas familias de clase media eligen colegios subvencionados para evitar el contacto con niños pobres, para dar a sus hijos un ambiente seguro y disciplinado y para que estén expuestos a los valores que ellos consideran importantes. Los padres pobres disponen de redes sociales más débiles y muchas veces descartan la opción de asistir a los colegios subvencionados por su costo, distancia desde la casa, o porque tienen miedo de que sus hijos pueden sufrir discriminación. Los padres de los dos grupos usan información recibida de sus contactos personales y la observación directa de los colegios para llegar a sus decisiones. La información oficial sobre el rendimiento académico de los colegios tiene poca influencia sobre su opción final.

Flores y Carrasco (2013) destacan los efectos de la oferta local de colegios en las decisiones de los padres. Como los padres pobres tienden a enviar a sus hijos a colegios locales y los colegios con mejores resultados están ubicados principalmente en barrios habitados por grupos socioeconómicos más acomodados, los padres pobres tienen peores opciones en su ámbito local. En contraste con la literatura que se enfoca en cómo los padres de clase media reproducen la segregación a través de sus decisiones, esta investigación muestra que todos los padres desean que sus hijos estudien en colegios con un buen rendimiento académico, pero la oferta local de colegios impide que los padres pobres pueden acceder a colegios con una oferta académica de calidad (véase también Chumacero et al., 2011; Thieme \& Treviño, 2013).

Esta investigación intenta integrar las perspectivas que se enfocan en los efectos que tienen la demanda de los padres de clase media y la oferta local de colegios, sobre la segregación educacional. En distintas zonas de Santiago, los padres de clase media alta buscan colegios cuyos alumnos también sean de clase media y así contribuyen a la reproducción de la segregación socioeconómica. A su vez, la oferta local de colegios afecta las opciones abiertas a los padres. En comunas cuya población es mayoritariamente de clase media alta, la mayoría de los colegios son particulares y los padres de estos grupos descartan los establecimientos subvencionados o municipales. A contrario, en comunas que tienen un porcentaje más alto de población trabajadora y pobre, las principales opciones disponibles para familias de clase media son colegios subvencionados. En ese caso, los padres de clase media baja eligen colegios subvencionados para evitar que sus hijos estudien en colegios municipales. A través de este análisis es posible visualizar cómo la segregación residencial influye en la oferta local de colegios y, a su vez, cómo los padres contribuyen a la reproducción de la segregación educacional a través de sus decisiones en el mercado educativo.

\section{Diseńo metodológico}

Este artículo forma parte de una investigación más amplia que intenta entender el accionar de adultos de distintas fracciones de las clases medias en los campos de la vivienda, la educación, la decoración del domicilio y el uso del tiempo libre (Stillerman, 2010). Con este objetivo general, se decidió seleccionar colegios tradicionales católicos y laicos alternativos en las comunas de Nunnoa y La Florida. Se las 
eligió porque Nunnoa es conocida en Santiago como la comuna prototípica de la clase media tradicional, que incluye a profesores, artistas y empleados públicos. Cuenta con una gran infraestructura de establecimientos educacionales e instituciones públicas y culturales (sedes de la Universidad de Chile y de la Pontificia Universidad Católica de Chile, el Centro Cultural de Nunñoa, el Estadio Nacional, el Teatro de la Universidad Católica, el Instituto de Salud Pública, colegios tradicionales, parques, y un patrimonio arquitectónico importante). A fines del siglo XIX, la comuna fue un importante lugar de residencia para empresarios inmigrantes judíos y árabes, que fueron rechazados por la elite tradicional durante largas décadas. Nuńoa tiene una población infantil (de 0 a 14 ańos) de 20.886 y cuenta con 22 colegios particulares, 11 colegios municipales y 15 colegios subvencionados (Biblioteca del Congreso Nacional de Chile, 2015a; Ministerio de Educación, Chile, 2015a). En Nunnoa, la distribución de los grupos socioeconómicos es el siguiente: $\mathrm{ABC} 1: 28,7 \%$; $\mathrm{C} 2$ : 35,1\%; C3: 20\%; D: 14,5\%; y E: 2\% (Adimark, 2004).

La Florida ha llegado a ser un importante centro de una clase media "emergente" a raíz de su desarrollo inmobiliario y comercial desde los fines de los años setenta. No cuenta con una infraestructura cultural y educativa tan desarrollada como Nunnoa; sin embargo, el sector privado ha intentado llenar ese vacío (particularmente la empresa Mall Plaza) construyendo un museo, un teatro, cines y un centro de espectáculos culturales. Además, aunque en su mayoría los colegios son de reciente formación, la comuna incluye colegios reconocidos, como el Instituto Lasalle, el Colegio Giordano Bruno y el Colegio Santa Cecilia. La Florida tiene una población infantil (de 0 a 14 años) de 73.449 y cuenta con 10 colegios particulares, 25 colegios municipales y 94 colegios subvencionados (Biblioteca del Congreso Nacional de Chile, 2015b; Ministerio de Educación, Chile, 2015b). En La Florida, la distribución de los grupos socioeconómicos es la siguiente: ABC1: 11,7\%; C2: 25\%; C3: 26,5\%; D: 30,5\%; y E: 6,2\% (Adimark, 2004). La mayoría de los entrevistados de las dos comunas pertenece a la clase media alta, esto es, grupos socioeconómicos (GSE) C1 y C2 (véase Asociación de Investigadores de Mercado [AIM], 2008; Barozet \& Fierro, 2011).

La elección de estas dos comunas permite un contraste interesante entre segmentos de las clases medias divididos en términos verticales (hay un porcentaje más grande de residentes de la clase media alta en Nuñoa, y segmentos más grandes de la clase media baja, trabajadora y pobre, en La Florida). Además, es razonable pensar que haya una diferenciación horizontal importante entre los habitantes de las dos comunas, con residentes de Nuñoa con mayor capital cultural que los residentes de La Florida.

La selección de los colegios para la muestra tuvo la siguiente lógica. Antes de elegir los establecimientos con que se trabajaría, se hizo una muestra de "bola de nieve" en las dos comunas, de manera de desarrollar un conocimiento preliminar del ambiente. La idea inicial del investigador fue comparar un colegio tradicional católico con uno laico alternativo (ambos mixtos) en cada comuna, para ver las diferencias sociodemográficas y culturales entre los apoderados en ambos tipos de instituciones. Se evitó estudiar colegios de "elite" porque se estimó que atraen a una población de clase alta y, además, son bastante anómalos en relación con la mayoría de los colegios. 
Después de esta etapa exploratoria, se eligió enfocar el estudio en el Liceo Experimental Manuel de Salas (LMS) y el Colegio Calasanz en Nunnoa, ambos relativamente antiguos y ligados a la identidad de la comuna. Además, están ubicados en el mismo sector, lo que permitía plantear la hipótesis de que muchos de los apoderados de ambos establecimientos viven en la misma zona, hecho que se dio en algunos casos. Los dos colegios son particulares.

En La Florida se llegó a tres colegios: uno tradicional católico, Rosario Concha (RC), y dos alternativos, Raimapu y Raíces Altazor (RA). Los tres están ubicados relativamente cercanos entre sí y, por tanto, muchos de los apoderados viven en lugares aledaños. Rosario Concha depende del Arzobispado de Santiago y es el más antiguo de los tres. Raimapu fue formado en los años ochenta por profesores despedidos de otro colegio y acogió a opositores al gobierno militar. Raíces Altazor fue formado por exprofesores de Raimapu, que buscaban un ambiente más progresista. Los tres colegios partieron como establecimientos particulares y posteriormente, por necesidades financieras, se convirtieron en colegios particulares subvencionados.

Se realizaron 68 entrevistas con 77 individuos (en varios casos se entrevistó a los dos miembros de una pareja) de 36 a 59 años de edad (con una edad promedia de 45 años), distribuidos de la siguiente forma: 12 entrevistas con 16 individuos en Calasanz, 14 entrevistas con 16 individuos en LMs, 6 entrevistas con 8 personas en RA, 6 entrevistas con 8 personas en Raimapu, 8 entrevistas con 10 individuos en RC, 7 entrevistas con apoderados de varios colegios en La Florida, y 9 entrevistas con apoderados de distintos colegios en Nunnoa y alrededores. Además, el autor entrevistó al orientador o al director de cada colegio. Con el objetivo de hacer una comparación lo más equilibrada posible, no se utilizarán las entrevistas de la muestra de bola de nieve, porque se realizó entre una y tres entrevistas en cada colegio. Además de las entrevistas, se hizo observación participante en actividades de colegio de LMS, Calasanz, RC y Raimapu, y en la casa de vacaciones de un apoderado de LMS.

\section{Familias de las clases medias y la elección de colegios en Nuuñoa y La Florida}

En esta sección se desarrollan los resultados de la investigación empírica. Como se describe en la literatura, los individuos de clase media alta de las dos comunas usan criterios similares para elegir un colegio para sus hijos. Buscan establecimientos que permitan el desarrollo integral de sus hijos, los resultados de la prueba SIMCE no representan un factor prioritario para su elección, quieren un colegio que entregue valores a sus hijos, y buscan uno cuyos alumnos sean de clase media. Hay diferencias en relación con el tipo de valores que quieren que el colegio imparta a sus hijos (los apoderados de colegios alternativos buscan un colegio laico que permita el desarrollo personal de sus hijos; los apoderados de colegios católicos buscan un "sello personal", una formación ética y un enfoque en el orden y la disciplina). Los miembros de la clase media baja se enfocan menos en la propuesta pedagógica del colegio de su hijo y no hablan en detalle sobre valores. Buscan un colegio seguro, con disciplina, y que proteja a sus hijos de las influencias de las drogas, el sexo y el alcohol. Estas disposiciones también se alinean con los resultados de muchas investigaciones. 
Las diferencias entre las dos comunas están relacionadas con la oferta local de los colegios y la composición socioeconómica de cada comuna. En Ñuñoa, con pocas excepciones, los padres apenas mencionan los colegios municipales o subvencionados como opciones para sus hijos: hacen su elección dentro de la gama de colegios particulares. Varios de ellos son exalumnos de ese tipo de establecimientos. Además, hacen un contraste entre su preferencia por una educación integral para sus hijos y otros padres "exitistas" o elitistas. Ellos valoran su propio capital cultural y rechazan a familias de elite o familias de clase media con menos capital cultural.

En contraste, los padres de La Florida eligen colegios subvencionados (por su gran oferta en la comuna) y hacen un contraste entre sus opciones y las de familias de clase media baja o familias pobres. Los padres con alto capital cultural se sienten incómodos con la llegada a su colegio de familias con menos capital cultural, o familias similares que envían a sus hijos a colegios particulares. Algunos de ellos rechazan los colegios municipales por su bajo rendimiento académico. Los padres de clase media baja prefieren los colegios subvencionados a los colegios municipales, por el temor de que sus hijos se vean afectados por niños con "malas costumbres" en los colegios municipales.

\section{Eligiendo colegios particulares en Nuñoa}

En la primera etapa de la búsqueda de colegios, los padres eliminan aquellos que, por distintos motivos, les parecen inaceptables. Los factores que influyen en esta decisión son las preocupaciones de los padres por el rendimiento académico, la seguridad, la distancia de los colegios a la casa, sus valores religiosos y educativos, y el costo de los colegios. Estos factores son similares entre las distintas fracciones de la clase media, pero los padres con más capital cultural y económico muestran mayor flexibilidad en relación con los temas del costo y distancia (Ball et al., 1995; Raczynski et al., 2010).

La religión es un factor que incide en el rechazo de ciertos colegios. Ledda, una kinesióloga, comenta: "Yo me había formado en un colegio católico, y me parece que es una ayuda en términos de los valores. No quería que mis hijas anduvieran muy alternativas, por ejemplo, con colores, con el pelo rojo" (entrevista, 24 de marzo de 2009) $)^{2}$. Otros padres descartaron los colegios religiosos. José, un arquitecto, recuerda: "Los colegios de curas me complican. Yo soy absolutamente laico y quiero permanecer asi”" (entrevista, 12 de noviembre de 2008).

El costo de algunos colegios los hizo inalcanzable para varias familias. Ernesto, un analista financiero, recuerda: "Ahora, el Kent lo desechamos porque en su momento nos pareció muy caro en la perspectiva de tener otro hijo" (entrevista, 13 de marzo de 2009). Los entrevistados también evitaron los colegios de "elite". Jimena, una analista en programación, comenta: "Pero yo creo que es lo mismo que te pasa con los colegios como el Andrée. Uno dice, 'Pucha, la formación humana es un cero a la izquierda', pero educacionalmente los gallos salen top. Todos en la universidad, todos top, top, top, con 'n' cantidad de influencias para después encontrar pega. 
Pero el corazón medio chiquitito. Si yo quisiera que mis hijas fueran exitistas, no las hubiese metido en el Manuel de Salas" (entrevista, 24 de noviembre de 2008).

Los entrevistados en Nuñoa descartan los colegios municipales como opción para sus hijos. Tatiana, una químico-farmacéutica, comenta: "Ahora, sí, podríamos haber puesto a las niñas en el Liceo 1 si la educación pública fuera lo que fue en mi época. Yo podía circular por Teatinos, por el centro, tranquilamente. No me gustaría que mis hijas circularan por el centro solas. Los recursos del Liceo 1 no son lo que eran, entonces ya para mí no es una opción" (entrevista, 14 de abril de 2009). Claudia, una psicóloga, plantea: "Lo que estoy pagando ahí, no podría pagar más, y ya para irme a otro lugar tendría que pagar muchísimo más que eso o decidirme por una educación subvencionada. Tampoco estoy de acuerdo con ese concepto de educación" (entrevista, 18 de noviembre de 2008). En contraste, los orientadores de los dos colegios ven los colegios municipales como una competencia. Carlos, el orientador de Calasanz, comenta: "Un colegio como el República de Siria que le vaya bien, pasa a ser muy atractivo para muchas familias. Si yo logro buenos resultados con mi hijo gratis versus un millón setecientos mil pesos al año, cualquiera lo piensa. El único 'plus' que tenemos nosotros es lo valórico" (entrevista, 6 de abril de 2009). Aunque los entrevistados de esta investigación rechazaron los colegios municipales, es probable que padres de la clase media baja vean a los colegios municipales exitosos como una buena opción para sus hijos.

En cuanto a los motivos por los que los padres de Núñoa seleccionaron un colegio para sus hijos, varios de ellos los enviaron al establecimiento al que ellos habían asisitido. Los exalumnos de LMS destacan la opción de quedar vinculados a un colegio laico, pluralista y tolerante, a pesar de reconocer que sus resultados académicos han empeorado. Además, enviar a sus hijos a LMs les permite mantener sus vínculos de amistad con otros exalumnos. El orientador del colegio llamó este fenómeno "el síndrome del exalumno: creen que pueden revivir su infancia" (entrevista, 7 de abril de 2009). La elección de LMs refleja las lealtades sociales y principios de los padres. Su opción también puede ser considerada como una estrategia (consciente o inconsciente) para enriquecer su fondo de capital social.

Algunos exalumnos de Calasanz vuelven con sus hijos al colegio (o a la cadena de los colegios escolapios en el caso de padres que asistieron al Colegio Hispanoamericano), buscando mantenerlos en un establecimiento "sano" donde se enseñan valores y disciplina, y donde hay una pequeña comunidad que acoge, ubica y protege a los niños y niñas. Marcelo, un profesor, comenta: "Lo que pasa es que el colegio se buscó primero porque somos exalumnos” (entrevista, 23 de marzo de 2009).

La mayoría de los apoderados de Nuńoa tienen una visión muy elaborada del mercado de la educación, los costos y beneficios de distintos proyectos educativos, y lo que buscan en un colegio. Hay importantes divisiones horizontales entre los apoderados de colegios laicos alternativos y los de colegios tradicionales católicos. Esta división se puede explicar a partir de las actividades profesionales de cada grupo. La mayoría de los padres en los colegios alternativos trabajan como educadores, funcionarios públicos calificados, o investigadores. Los apoderados en los colegios católicos trabajan en el sector privado en contabilidad, análisis financiero, 
informática, o como abogados o médicos. Sin embargo, los dos grupos comparten el deseo de tener un colegio que entregue valores a sus hijos.

Los apoderados de LMs buscan un colegio laico, antiautoritario, tolerante, integrador, y que promueva el desarrollo individual y la independencia de sus hijos. En su mayoría critican la reforma educativa de 1981 y expresan dudas sobre el valor de los parámetros usados para evaluar los colegios. Evitan presionar a sus hijos por las notas, especialmente en la enseñanza básica, y quieren promover su creatividad e iniciativa. Alicia, una investigadora en el campo de la salud, comenta: "Víctor está estudiando piano porque está en el Manuel de Salas, porque lo incentivaron: como que lo descubrieron" (entrevista, 10 de noviembre de 2008). Estos padres tienen inquietudes intelectuales y políticas que quieren transmitir a sus hijos a través del colegio. Además, buscan un colegio donde los niños y sus padres encuentran una diversidad de opiniones. Tal como plantean Raveauda y Van Zanten (2007), intentan cumplir sus deseos de ser "buenos padres" y "buenos ciudadanos".

Los apoderados Calasanz buscan un colegio con buenos resultados académicos que enfatice el orden, la disciplina, y un ambiente acogedor y sano. Muestran preocupación por las drogas, la sexualidad y la delincuencia, y valorizan talleres y pastorales que ayuden a los apoderados a manejar estos temas con sus hijos. Mientras expresan más interés en "los resultados" que los apoderados de LMs, critican el enfoque exclusivo en "los números". Claudia, una abogada, comenta: "Lo primero que nos importaba era que fuera un colegio católico, que tuviera un sello personal: que los niños sean educados en la fe, pero como con un sello especial, de ser solidarios, de ser como niños sencillos. En segundo lugar estaba el tema académico: si el colegio no les daba todos los recursos necesarios, yo se los podía dar" (entrevista, 13 de marzo de 2009). Como indica Claudia, varios padres de clase media alta en las dos comunas comentaron que ellos mismos podrían suplir las debilidades académicas de su colegio o contratar profesores privados. Esta opción solo es factible para padres con niveles altos de capital cultural y económico.

Las familias de clase media eligen colegios cuyos estudiantes son de la misma clase (Ball et al., 1995; Raczynski et al., 2010; Thieme \& Treviño, 2013). Sin embargo, en todos los colegios estudiados se dan relaciones entre las distintas clases sociales y/o distintas fracciones de las clases medias. Dentro de este contexto, los padres hablan de distintas formas sobre los "otros" de los cuales quieren distinguirse (Méndez, 2008; Méndez \& Barozet, 2012; Sabatini et al., 2012).

Los apoderados de LMs hablan en forma indirecta sobre los "otros" que habitan su colegio. Como son personas de izquierda, les parece que sería mal visto criticar a personas con menos educación que ellos. Por tanto, su incomodidad con los "otros" se expresa en relación con los valores del colegio. Leonardo, un arquitecto, comenta: "El Manuel de Salas tiene un historial que es importante. Era un colegio experimental. Fue la primera parte en que se generó la autodisciplina en los niños. Los papás jóvenes de la escuela básica no tienen idea, po' " (entrevista, 14 de abril de 2009). Los apoderados de Calasanz critican las malas costumbres de los padres más competitivos. Javiera, una secretaria, plantea: "Algunas veces pienso que los apoderados son súper falta de respeto en los estacionamientos, y en actitudes que tienen con los mismos apoderados. Tú decí, 'Pucha, si somos todos de lo mismo'. Pa' mí es 
falta de educación. A veces hay gente que están preocupadas más de las apariencias que de educación" (entrevista, 11 de marzo de 2009).

\section{Eligiendo colegios subvencionados en La Florida}

Igual que los padres de Nunńoa, los apoderados de los colegios de La Florida rechazaron varios colegios por motivos religiosos. Elba, una educadora de párvulos, dice: "No me interesa mucho la religión; a mi marido más, porque él se educó en colegio católico, y él quería valores" (entrevista, 4 de mayo de 2010). Iván, un historiador y planificador regional, comenta: "Lo primero fue investigar y hacer el catastro de todos los colegios, y descartar todos los colegios religiosos" (entrevista, 27 de mayo de 2009).

Todos los entrevistados descartaron colegios municipales, aunque algunos de ellos los consideraron en un primer momento. Iván, citado más arriba, plantea: "Miramos los colegios municipales, los descartamos también por el rendimiento, el SIMCE y todo, y características de nuestra hija. Lo que pasa es que nuestra hija es 'fundida' [regalona]. Ella era más bien tímida y por una cuestión pedagógica no la veíamos en un curso de cuarenta y cinco cabros. Ya, descartaste los colegios municipales". Álvaro, un ejecutivo de ventas, comenta: "Siempre andábamos buscando un tipo que no sea municipal, porque no es lo mismo lo municipal que antiguamente, cuando nosotros estudiábamos, con lo que hoy en día es" (entrevista, 26 de abril de 2010). Mario, un profesional en computación, nota la contradicción entre sus valores y su rechazo a los colegios municipales: "Andrea se ha preguntado: qué imagen da que la jefa de Educación de la Municipalidad tenga a sus hijos en un colegio particular subvencionado [ríe]. Claro, es una tremenda contradicción. Yo digo, 'bueno, quizás a lo que nosotros aspiramos es que todos los colegios sean como el Raimapu y no como los otros'” (entrevista, 29 de abril de 2009).

En Núñoa los entrevistados rechazaron los colegios de elite, pero en La Florida descartan los colegios particulares, por su precepción de que su rendimiento académico es débil y los apoderados tienen poco capital cultural. José Miguel, un químico, comenta: "Aquí en el British American School no hablan inglés los niños. [Ríe] Tienen el nombre, como que lo hace tener un estatus más alto. Es como si fueran en el Grange [un colegio de elite]. En Chile somos muy abocados a tratar de aparentar más de lo que realmente somos" (entrevista, 26 de mayo de 2009). Mario, citado más arriba, comenta: "Antes que compráramos esta casa, vivimos al frente del British High School. Ay, pero era un desastre. Era un ata'o de bestias en la mañana que llegaban ahí a tirar a los cabros chicos. Claramente no era eso lo que queríamos para nuestros hijos".

La mayoría de los entrevistados depende de sus contactos personales para conseguir información confiable sobre los colegios (Raczynski, 2010; Ball \& Vincent, 1998). Hubo diferencias en el capital social del que disponían los entrevistados según su posición socioeconómica. Iván, citado más arriba, recuerda: "Yo me asesoré con una amiga mía que supervisaba prácticas en el Pedagógico y, por lo tanto, ella iba a supervisar en la zona suroriente. Vive aquí en La Florida. Me tiró una lista de como seis, descartamos un par, al final nos quedamos con como cuatro" (entrevista, 27 de mayo del 2009). Otros individuos de clase media alta conocían a profesores, a otros profesionales o a egresados del colegio que eligieron. Algunos 
entrevistados tuvieron a familiares con hijos en el colegio que seleccionaron; otros padres eligieron el colegio donde la madre es profesora.

Los entrevistados de clase media baja dependieron de recomendaciones de sus amigos o familiares, o simplemente buscaron cerca de su casa. Paola, una secretaria, comenta: "Yo estuve averiguando sobre colegios que estuviesen como al alcance de poder cancelarlo nosotros, que no fuera ni el más barato ni el más caro, y llegamos al Rosario, po' " (entrevista, 16 de junio del 2009). Aunque los padres de La Florida no son exalumnos de colegios del sector, algunos desearon rescatar algunos aspectos del colegio de su infancia. Ana, una secretaria y especialista en relaciones públicas que estudió en un colegio católico, comenta: "Me gustó el colegio porque era católico; y además, era más chiquitito” (entrevista, 15 de junio de 2009).

Los padres de clase media alta buscaron, en el colegio que eligieron, elementos similares a los que aspiraban sus contrapartes en Nuñoa. Los padres de colegios alternativos buscaron un colegio que promoviera la creatividad y el debate. Tomás, un ingeniero y apoderado de RA, comenta: "Los niños que salen de ahí son críticos, son muy autónomos, son muy sanos en sus juicios o en sus opiniones" (entrevista, 15 de mayo de 2009). Los apoderados de clase media alta de RC también tienen objetivos similares a aquellos de los apoderados de Calasanz. Nelson, un contador, comenta: "Lo cristiano-occidental del colegio les da un cierto marco occidental de cultura, de educación, de ética" (entrevista, 16 de junio de 2009).

Los apoderados de la clase media baja tienen un discurso más pragmático en relación con su elección de colegio. Destacan las buenas impresiones que tuvieron del director o directora del colegio, las amistades de sus hijos con niños del establecimiento, la disponibilidad de becas, la cercanía a la casa, la disciplina escolar o el precio. Tienen un discurso menos elaborado que los padres de clase media alta en relación con el tipo de educación que buscan. Por ejemplo, Marcela, una chofer de camiones, comenta: "Me cambié al Rosario porque todavía es un colegio como más estricto, que exige, que tiene un poco más de disciplina, y es lo que me alcanza para pagar" (entrevista, 27 de abril de 2010).

En La Florida, los padres se preocupan de los "otros" que enfrentan en el colegio, pero las características socioeconómicas de la comuna crean una dinámica distinta de la observada en Núñoa. José Miguel, citado más arriba, comenta: "Antes de la crisis había como una cierta estabilidad económica, pero después el dinero se volatilizó mucho: hubo mucho IPC y mucha pérdida de trabajo. Entonces, mucha gente tuvo que retirar a sus hijos de los colegios particulares. Por eso se hicieron subvencionados, y bajaron y pudieron crecer también en la cantidad de alumnos". La conversión de Raimapu a un colegio subvencionado, además de provocar una fuga de algunos apoderados que no estuvieron de acuerdo con la decisión, permitió la entrada de familias con menos capital cultural y económico. Alejandro, un profesor, dice: "Pero hay conjuntos familiares que están entrando en este otro ritmo más consumista, más competitivo, más exitista, en donde ya les interesa más la parte cognitiva y empiezan a despreciar más la otra parte social, afectiva" (entrevista, 13 de mayo de 2009). Elba, citada más arriba, nota diferencias similares en RC: "Aquí hay un grupo de apoderados. Creo que para ellos es harta plata y la utilizan en comprar ropas, el mejor celular y zapatillas de ochenta mil pesos. Mi plata es para 
otra cosa. La gente no se preocupa de educar: que el nińo tenga experiencias que le puedan dar una visión distinta de la vida. No compran libros".

En contraste, los padres de clase media baja evitan el contacto con familias pobres. Paola, citada más arriba, comenta: "Claro, porque si yo pongo a mi hijo en un colegio municipalizado donde "sha" y "ah", esas costumbres lamentablemente se pegan. Uno tiene que siempre tratar de ir en la misma línea de uno o más arriba, pero no más abajo, po’... si la cosa es superación”. Marcela, citada más arriba, dice: "Aquí hay municipalizados y hay subvencionados, pero malos, po'. Tendría que a los niños enseñarles a usar pistola, o un cuchillo. Entonces se hace un esfuerzo sobrehumano para que estén en un colegio particular subvencionado, que es lo que uno puede pagar".

\section{Conclusiones}

Esta investigación ha mostrado que los padres de clase media en Santiago utilizan su capital social, económico y cultural para elegir un colegio que sea lo más adecuado posible para sus hijos y que represente sus valores. Como la distribución de estos capitales no es igual a través de la población, estas estrategias dan ventajas a las familias de la clase media alta para elegir colegios de mejor calidad, por sobre familias de la clase media baja. Además, la oferta local de educación a nivel comunal tiene un efecto importante en las opciones de los padres. En Nuńoa, la gran cantidad de colegios particulares pagados influye en la decisión de los padres de clase media alta de descartar los colegios municipales y los particulares subvencionados. Además, la concentración de población acomodada en la comuna tiene como resultado que estos padres se comparen con familias de elite y de la clase media baja, pero para ellos los pobres son invisibles en el ámbito educativo. En La Florida, la gran cantidad de colegios subvencionados y el porcentaje más alto de los GSE C3, D y E crea una dinámica distinta. Padres de clase media alta eligen colegios subvencionados y se comparan con familias con menos capital cultural, mientras los apoderados de la clase media baja se alejan de las familias pobres. En las dos comunas, las estrategias de los padres y la oferta de colegios se combinan para reproducir la segregación educacional y reforzar el empobrecimiento de los colegios municipales.

Estos resultados constituyen un aporte a las discusiones sobre la segregación residencial y educacional. Concuerdan con Sabatini et al. (2012) en que a los padres de clase media les cuesta enviar a sus hijos a un colegio con niños pobres. En contraste con Rasse (2015), quien encontró que la integración social entre adultos de clase media y pobres es más factible cuando la brecha económica entre ellos no es tan grande, el hecho de que los apoderados de clase media baja rechacen a los pobres muestra que los grupos socioeconómicos que tienen salarios no tan diferentes pueden evitar integrarse. Sin embargo, los resultados concuerdan con su planteamiento respecto de que la falta de seguridad puede ser un obstáculo para la integración residencial.

Esta investigación también hace una síntesis entre estudios sobre educación enfocados en las estrategias de los padres, y otros centrados en la oferta de colegios, ambos grupos como fuentes de la segregación educacional. Los resultados 
concuerdan con Thieme y Treviño (2013) y Flores y Carrasco (2013), quienes plantean que la oferta local de colegios tiene efectos importantes en la segregación educacional. Sin embargo, queda en evidencia que las opciones de los apoderados de evitar los colegios municipales en las dos comunas también contribuyen a la reproducción de la segregación educacional.

Por último, esta investigación muestra cómo, en Chile, miembros de distintas fracciones de la clase media tienen actitudes ambivalentes hacia la desigualdad. Buscan distintas maneras de aislar a sus hijos tanto de miembros de "la clase media emergente", que valoriza la competencia y el éxito, como de los pobres y de las elites. Además, muestra las tensiones que experimentan entre sus deseos de ser "buenos padres" y "buenos ciudadanos" (Raveauda \& Van Zanten, 2007). Estos individuos revelan la ambigüedad de su condición privilegiada en relación con los pobres y su exclusión de la elite. Los padres de clase media tienen distintas capacidades de navegar el mercado de la educación, pero a la vez expresan su malestar por los defectos y los dilemas que se les presentan en ese mercado.

\section{Referencias bibliográficas}

Adimark. 2004. Mapa socioeconómico de Chile. Santiago, Chile: Adimark.

Alves, F., Elacqua, G., Koslinki, M., Martínez, M., Santos, H. \& Urbina, D. (2015). Winners and losers of school choice: Evidence from Rio de Janeiro, Brazil and Santiago, Chile. International Journal of Educational Development, 41(C), 25-34.

Ariztía, T. (2009). Arreglando la casa propia: la cultura material de la movilidad social. En M.Tironi \& F. Pérez (Eds.), sCL: espacios, prácticas y cultura urbana (pp. 70-85). Serie Teoría y Obra Vol. 9. Santiago: Ediciones Arq, Escuela de Arquitectura, Pontificia Universidad Católica de Chile.

Asociación de Investigadores de Mercado (AIM). (2008). Grupos socioeconómicos. Santiago, Chile: Asociación Chilena de Empresas de Investigación de Mercado. Disponible en http://www.aimchile.cl/wp-content/uploads/2011/12/Grupos_Socioeconomicos_ AIM-2008.pdf

Ball, S. J. \& Vincent, C. (1998). I heard it on the grapevine: Hot knowledge and school choice. British Journal of Sociology of Education, 19(3), 377-400. doi: 10.1080/0142569980190307

Ball, S. J. (2003). Class strategies and the education market. Londres: RoutledgeFalmer.

Ball, S. J., Bowe, R. \& Gewirtz, S. (1995). Circuits of schooling: A sociological exploration of parental choice of school in social class contexts. The Sociological Review, 43(1), 52-78. doi: 10.1111/j.1467-954X.1995.tb02478.x

Barozet, E. \& Fierro, J. (2011). The middle class in Chile: The characteristics and evolution 1990-2011. Kas International Reports, (12), 25-41. Disponible en http://www.kas.de/ wf/en/33.29622/ 
Barozet, E. (2006). El valor histórico del pituto: clase media, integración y diferenciación social en Chile. Revista de Sociología del Departamento de Sociología de la Universidad de Chile, (20), 69-96. Disponible en http://www2.facso.uchile.cl/sociologia/docs/ Barozet_ValorHistorico_Pituto.pdf

Biblioteca del Congreso Nacional de Chile (2015a). Población por grupos de edad 2002 y proyectada 2012 INE. http://reportescomunales.bcn.cl/2012/index. php/\%C3\%91u\%C3\%B1oa\#Poblaci.C3.B3n_por_grupos_de_edad_2002_y_ proyectada_2012_INE

Biblioteca del Congreso Nacional de Chile (2015b). Población por grupos de edad 2002 y proyectada 2012 INE. http://reportescomunales.bcn.cl/2012/index.php/La Florida\#Poblaci.C3.B3n_por_grupos_de_edad_2002_y_proyectada_2012_INE (accedido el 16 de agosto del 2015).

Bourdieu, P. \& Passeron, J. C. (1990). Reproduction in education, society and culture. (2 $2^{\text {nd }}$ ed.). Londres: Sage.

Bourdieu, P. (1984). Distinction. Cambridge, Ma: Harvard University Press.

Bourdieu, P. (1997). The forms of capital. En A.H. Halsey, H. Lauder, P. Brown \& A. Stuart Wells (Eds.), Education, culture, economy and society (pp. 46-58). Nueva York: Oxford University Press.

Chumacero, R. A., Gómez, D. \& Paredes, R. D. (2011). I would walk 500 miles (if it paid): Vouchers and school choice in Chile. Economics of Education Review, 30(5), 11031114. http://dx.doi.org/10.1016/j.econedurev.2011.05.015

Contreras, D., Sepúlveda, P. \& Barros, S. (2010). When schools are the ones that choose: The effects of screening in Chile. Social Science Quarterly, 91(5), 1349-1368. doi: 10.1111/j.1540-6237.2010.00735.x

Elacqua, G. \& Santos, H. (2013). Preferencias reveladas de los proveedores de educación privada en Chile: el caso de la Ley de Subvención Escolar Preferencial. Gestión y Política Pública, 22(1), 85-129. Disponible en http://www.redalyc.org/articulo. oa?id $=13325600003$

Flores, C. \& Carrasco, A. (2013). (Des)igualdad de oportunidades para elegir escuelas: preferencias, libertad de elección y segregación escolar. Documento de Referencia 2. Espacio Público. Disponible en http://espaciopublico.cl/publicaciones/detalle.tpl?id=8

Lareau, A. 2003. Unequal childhoods: Class, race, and family life. Berkeley, cA: University of California Press.

León, A. \& Martínez, J. (2007). La estratificación social en Chile a fines del siglo xx. En R.Franco, A. León \& R. Atria (Coords.), Estratificación y movilidad social en América Latina: transformaciones estructurales de un cuarto de siglo (pp. 303-338). Santiago: LOM / Cepal / GTZ.

Márquez, F. \& Pérez, F. (2008). Spatial frontiers and neo-communitarian identities in the city: The case of Santiago de Chile. Urban Studies, 45(7), 1461-1483. doi: 10.1177/0042098008090684

Méndez, M. L. \& Barozet, E. (2012). Lo auténtico también es público. Comprensión de lo público desde las clases medias en Chile. Polis, 11(31), 2-15. http://dx.doi. org/10.4067/S0718-65682012000100011 
Méndez, M. L. (2008). Middle class identities in a neoliberal age: tensions between contested authenticities. The Sociological Review, 56(2), 220-237. doi: 10.1111/j.1467954X.2008.00785.x

Ministerio de Educación, Chile (2015a). Listado comunal: comuna Nunnoa. http://www.mime. mineduc.cl/mime-web/mvc/mime/listado (acceso el 16 de agosto de 2015).

Ministerio de Educación, Chile (2015b). Listado comunal: comuna La Florida. http://www. mime.mineduc.cl $/ \mathrm{mime}-\mathrm{web} / \mathrm{mvc} / \mathrm{mime} / \mathrm{listado}$ (acceso el 16 de agosto de 2015).

Mizala, A. \& Torche, F. (2012). Bringing the schools back in: the stratification of educational achievement in the Chilean voucher system. International Journal of Educational Development, 32(1), 132-144. doi: 10.1016/j.ijedudev.2010.09.004

Raczynski, D., Salinas, D., de la Fuente, L., Hernández, M. \& Lattz, M. (2010). Hacia una estrategia de validación de la educación pública-municipal: imaginarios, valoraciones y demandas de las familias. Informe Final Proyecto Fondo de Investigación y Desarrollo en Educación. Santiago: Gobierno de Chile, Departamento de Estudios y Desarrollo, División de Planificación y Finanzas, Ministerio de Educación. Disponible en http:// sgdce.mineduc.cl/descargar.php?id_doc $=201208211210430$

Rasse, A. (2015). Juntos pero no revueltos: procesos de integración social en fronteras residenciales entre hogares de distinto nivel socioeconómico. EURE, 41(122), 125143. http://dx.doi.org/10.4067/S0250-71612015000100006

Raveauda, M. \& van Zanten, A. (2007). Choosing the local school: middle class parents' values and social and ethnic mix in London and Paris. Journal of Education Policy, 22(1), $107-$ 124. doi: $10.1080 / 02680930601065817$

Sabatini, F. \& Cáceres, G. (2004). Los barrios cerrados y la ruptura del patrón tradicional de segregación en las ciudades latinoamericanas: el caso de Santiago de Chile. En G.Cáceres \& F. Sabatini (Eds.), Barrios cerrados en Santiago de Chile: entre la exclusion y la integración residencial (pp. 9-43). Santiago, Chile: Lincoln Institute of Land Policy/ Pontificia Universidad Católica de Chile, Instituto de Geografía.

Sabatini, F., Cáceres, G. \& Cerda, J. (2001). Segregación residencial en las principales ciudades chilenas: tendencias de las tres últimas décadas y posibles cursos de acción. EURE, 27(82), 21-42. http://dx.doi.org/10.4067/S0250-71612001008200002

Sabatini, F., Rasse, A., Mora, P. \& Brain, I. (2012). ¿ Es posible la integración residencial en las ciudades chilenas?: disposición de los grupos medios y altos a la integración con grupos de extracción popular. EURE, 38(115), 159-194. http://dx.doi.org/10.4067/ S0250-71612012000300008

Stillerman, J. (2010). The contested spaces of Chile's middle classes. Political Power and Social Theory, 21, 209-238. doi: 10.1108/S0198-8719(2010)0000021013

Thieme, C. \& Treviño, E. (2013). School choice and market imperfections: Evidence from Chile. Education and Urban Society, 45(6), 635-657. doi: 10.1177/0013124511413387

Vargas, Felipe. (2015). Presidenta Bachelet promulga ley que pone fin al lucro, copago y selección escolar. Emol.com - http://www.emol.com/noticias/nacional/2015/05/29/719135/ presidenta-bachelet-firma-promulgacion-de-ley-que-pone-fin-al-lucro-copago-yseleccion.html (acceso el 28 de febrero de 2016). 